\title{
Nobel Lecture: Evading the Goldstone theorem ${ }^{*}$
}

\author{
Peter W. Higgs \\ (published 3 July 2014)
}

\section{CONTENTS}

I. Broken Symmetries

851

II. The Goldstone Theorem

III. Can One Evade the Goldstone Theorem?

IV. How to Evade the Goldstone Theorem

V. Postscript

References

851

851

852

852

852

My story begins in 1960, when I was appointed Lecturer in Mathematical Physics at the University of Edinburgh. Before I took up my appointment I was invited to serve on the committee of the first Scottish Universities Summer School in Physics. I was asked to act as Steward at the school in July, my principal duty being to purchase and look after supplies of the wine which was to be served at dinner each evening.

The students at the school included four who stayed up late into the night in the common room of Newbattle Abbey College (the crypt of a former abbey) discussing theoretical physics and rarely got up in time for the first lecture of the following day. They were Dr. N. Cabibbo (Rome), Dr. S. L. Glashow (CERN), Mr. D. W. Robinson (Oxford), and Mr. M. J. G. Veltman (Utrecht). Many years later Cabibbo told me that their discussions had been lubricated by bottles of wine collected after dinner and hidden inside the grandfather clock in the crypt.

I did not take part in these discussions, since I had other things to do (such as conserving wine). Consequently, I did not learn about Glashow's paper (Glashow, 1961) on electroweak unification, which had already been written.

\section{BROKEN SYMMETRIES}

During my first year as a lecturer I was in search of a worthwhile research program. In the previous four years in London I had rather lost my way in particle physics and had become interested in quantum gravity. Symmetry had fascinated me since my student days, and I was puzzled by the approximate symmetries (what are now called flavor symmetries) of particle physics.

Then in 1961 I read Nambu's (Nambu, 1960) and Goldstone's (Goldstone, 1961) papers on models of symmetry breaking in particle physics based on an analogy with the theory of superconductivity. [Nambu's models were inspired by the Bardeen, Cooper, and Schrieffer theory (Bardeen, Cooper, and Schrieffer, 1957), based on Bose condensation of Cooper pairs of electrons: Goldstone used scalar fields, with a "wine bottle" potential to induce Bose condensation, as in the earlier Ginzburg-Landau theory.] What I found very attractive was the concept of a spontaneously broken symmetry, one that

*The 2013 Nobel Prize for Physics was shared by François Englert and Peter Higgs. These papers are the text of the address given in conjunction with the award. is exact in the underlying dynamics but appears broken in the observed phenomena as a consequence of an asymmetric ground state ("vacuum" in quantum field theory).

Most particle theorists at the time did not pay much attention to the ideas of Nambu and Goldstone. Quantum field theory was out of fashion, despite its success in quantum electrodynamics; it was failing to describe either the strong or the weak interactions.

Besides, condensed matter physics was commonly viewed as another country. At a Cornell seminar in 1960 Victor Weisskopf remarked (as recalled by Robert Brout)

"Particle physicists are so desperate these days that they have to borrow from the new things coming up in many body physics-like BCS. Perhaps something will come of it."

\section{THE GOLDSTONE THEOREM}

There was an obstacle to the success of the NambuGoldstone program. Nambu had shown how spontaneous breaking of a chiral symmetry could generate the masses of spin- $\frac{1}{2}$ particles, such as the proton and neutron, but his model predicted massless spin-0 particles (pions?), contrary to experimental evidence. (As noted by Weinberg any such particles would dominate the radiation of energy from stars.) Goldstone had argued that such massless particles would always be the result of excitations around the trough of the wine bottle potential.

In 1962 a paper entitled "Broken Symmetries" by Goldstone, Salam, and Weinberg (Goldstone, Salam, and Weinberg, 1962) proved the "Goldstone theorem," that "In a manifestly Lorentz-invariant quantum field theory, if there is a continuous symmetry under which the Lagrangian is invariant, then either the vacuum state is also invariant or there must exist spinless particles of zero mass."

This theorem appeared to put an end to Nambu's program.

\section{CAN ONE EVADE THE GOLDSTONE THEOREM?}

In 1963 the condensed matter theorist Phil Anderson (Anderson, 1962) pointed out that in a superconductor the Goldstone mode becomes a massive "plasmon" mode due to long-range (Coulomb) forces, and that this mode is just the longitudinal partner of transverse electromagnetic modes, which are also massive. Anderson remarked "The Goldstone zero-mass difficulty is not a serious one, because we can probably cancel it off against an equal Yang-Mills zero-mass problem." However, he did not show that there was a flaw in the Goldstone theorem and he did not discuss any relativistic model, so particle theorists such as myself received his remark with skepticism. 
In March 1964 Abe Klein and Ben Lee (Klein and Lee, 1964) suggested that, even in relativistic theories, a certain equation which was crucial for the proof of the Goldstone theorem could be modified by the addition of an extra term, just as in condensed matter theories. But in June Wally Gilbert (who was in transition from theoretical physics to molecular biology, for which he later won a Nobel Prize for Chemistry) ruled out this term as a violation of Lorentz invariance.

It was at this point that my intervention took place.

\section{HOW TO EVADE THE GOLDSTONE THEOREM}

I read Gilbert's paper (Gilbert, 1964) on 16 July 1964-it had been published a month earlier, but in those days the University of Edinburgh's copies of Physical Review Letters came by sea - and I was upset because it implied that there was no way to evade Goldstone's theorem. But over the following weekend I began to recall that I had seen similar apparent violations of Lorentz invariance elsewhere, in no less a theory than quantum electrodynamics, as formulated by Julian Schwinger.

Quantum electrodynamics is invariant under gauge transformations and the gauge must be fixed before well-defined quantum formalism can be set up. The fashionable way to do this was to choose a Lorentz gauge, which was manifestly compatible with relativity. However, such a gauge had unsatisfactory features that led Schwinger to prefer a Coulomb gauge, which introduces an apparent conflict with relativity. Nevertheless, it was well known that this choice did not lead to any conflict between the predicted physics and relativity.

Schwinger (Schwinger, 1962a, 1962b) had, as recently as 1962, written papers in which he demolished the folklore that it is gauge invariance alone that requires photons to be massless. He had provided examples of some properties of a gauge theory containing massive "photons," but without describing explicitly the underlying dynamics.

During the weekend of 18 and 19 July it occurred to me that Schwinger's way of formulating gauge theories undermined the axioms which had been used to prove the Goldstone theorem. So gauge theories might save Nambu's program.

During the following week I wrote a short paper about this. It was sent to Physics Letters on 24 July and was accepted for publication (Higgs, 1964a).

By then I had written down the (classical) field equations of the simplest illustrative model that I could imagine, the result of introducing an electromagnetic interaction into Goldstone's simplest scalar model. It became obvious that in this model the Goldstone massless mode became the longitudinal polarization of a massive spin-1 photon, just as Anderson had suggested.

My second short paper, consisting of a brief account of this model, was sent to Physics Letters on 31 July. It was rejected. The editor (at CERN) suggested that I develop my ideas further and write a full account for Il Nuovo Cimento.

I was indignant; it seemed that the referee had not seen the point of my paper. (Later, a colleague who returned from a month's visit to CERN told me that the theorists there did not think it had any relevance to particle physics.) Besides, it seemed odd that the earlier paper had been accepted but the more physical sequel had not.

I decided to augment the paper by some remarks on possible physical consequences and to send the revised version across the Atlantic to Physical Review Letters. Among the additional material was the remark, "It is worth noting that an essential feature of this type of theory is the prediction of incomplete multiplets of scalar and vector bosons."

The revised paper was received by Physical Review Letters on 31 August and was accepted (Higgs, 1964b). The referee invited me to comment on the relation of my paper to that of Englert and Brout (Englert and Brout, 1964), whose paper (received on 22 June) had been published that day. Until then I had been unaware of their work, but I added a footnote to my paper as soon as I had received a copy of theirs. Twenty years later, at a conference in 1984, I met Nambu, who revealed that he had refereed both papers.

\section{POSTSCRIPT}

It took some time for the work of Englert and Brout and myself [and of Guralnik, Hagen, and Kibble (Guralnik, Hagen, and Kibble, 1964), who published a little later] to gain acceptance.

My longer (Higgs, 1966) paper was written in autumn 1965 at Chapel Hill, North Carolina, where I was spending a sabbatical year at the invitation of Bryce DeWitt as a consequence of my interest in quantum gravity. A preprint sent to Freeman Dyson received a positive response; he invited me to give a talk at I. A. S. Princeton. There, in March 1966, I faced an audience including axiomatic quantum field theorists who still believed that there could be no exceptions to the Goldstone theorem.

The next day I gave a talk at Harvard (arranged by Stanley Deser) to another skeptical audience, including Wally Gilbert. I survived this too. After the seminar Shelly Glashow complimented me on having invented "a nice model," but he did not recognize its relevance to his electroweak theory-a missed opportunity!

Like Nambu, the six of us who published in 1964 expected to apply our ideas to the broken flavor symmetries of the strong interactions, but this did not work. So it was left to Weinberg (Weinberg, 1967) and Salam (Salam, 1968) in 1967 to find the right application.

Four more years passed before Gerard 't Hooft ('t Hooft, 1971), in an extension of Veltman's program, proved the renormalizability of such theories and another two before the discovery of weak neutral currents indicated that Glashow's electroweak unification was the correct one. And in 1976 Ellis, Gaillard, and Nanopoulos (Ellis, Gaillard, and Nanopoulos, 1976) at CERN encouraged experimentalists to look for the massive spinless boson that the theory predicted.

\section{REFERENCES}

Anderson, P. W., 1963, Phys. Rev. 130, 439.

Bardeen, J., L. N. Cooper, and J. R. Schrieffer, 1957, Phys. Rev. 106, 162. 
Ellis, John, Mary K. Gaillard, and D. V. Nanopoulos, 1976, Nucl. Phys. B106, 292.

Englert, F., and R. Brout, 1964, Phys. Rev. Lett. 13, 321.

Gilbert, Walter, 1964, Phys. Rev. Lett. 12, 713.

Glashow, S. L., 1961, Nucl. Phys. 22, 579.

Goldstone, J., 1961, Nuovo Cimento 19, 154.

Goldstone, J., A. Salam, and S. Weinberg, 1962, Phys. Rev. 127, 965.

Guralnik, G. S., C. R. Hagen, and T. W. B. Kibble, 1964, Phys. Rev. Lett. 13, 585.

Higgs, P. W., 1964a, Phys. Lett. 12, 132.
Higgs, P. W., 1964b, Phys. Rev. Lett. 13, 508.

Higgs, P. W., 1966, Phys. Rev. 145, 1156.

Klein, Abraham, and Benjamin W. Lee, 1964, Phys. Rev. Lett. 12, 266. Nambu, Y., 1960, Phys. Rev. Lett. 4, 380.

Salam, A., 1968, in Proceedings of the 8th Nobel Symposium (Almqvist and Wiksell, Stockholm), pp. 367-377.

Schwinger, Julian, 1962a, Phys. Rev. 125, 397.

Schwinger, Julian, 1962b, Phys. Rev. 128, 2425.

't Hooft, G., 1971, Nucl. Phys. B33, 173.

Weinberg, S., 1967, Phys. Rev. Lett. 19, 1264. 